Journal Club

Editor's Note: These short reviews of recent JNeurosci articles, written exclusively by students or postdoctoral fellows, summarize the important findings of the paper and provide additional insight and commentary. If the authors of the highlighted article have written a response to the Journal Club, the response can be found by viewing the Journal Club at www.jneurosci.org. For more information on the format, review process, and purpose of Journal Club articles, please see http://jneurosci.org/content/ preparing-manuscript\#journalclub.

\title{
How Attention Enhances Spatial Resolution: Preferential Gain Enhancement of High Spatial Frequency Neurons
}

\author{
Antoine Barbot \\ Flaum Eye Institute, University of Rochester Medical Center and Center for Visual Science, University of Rochester, Rochester, New York 14642 \\ Review of Mineault et al.
}

How we experience the world around us strongly depends on behavioral states. We are constantly confronted with an overwhelming amount of sensory input, much more than our brain, limited in resources, can process at a given instant. To efficiently manage limited resources, neural activity is tightly coupled to changes in waking state, such as variations in arousal and attention. However, the relation between behavioral states and cortical processing remains poorly understood.

Recent studies in mice have provided compelling evidence that cortical activity is highly state-dependent (Niell and Stryker, 2010; McGinley et al., 2015). Such studies use a deceptively basic index of arousal, pupil dilation, to monitor behavioral states in head-fixed mice. Pupillometry allows changes in arousal to be tracked between resting (low arousal, constricted pupil) and active (high arousal, dilated pupil) states (McGinley et al., 2015). During active locomotion, neurons in the mouse primary visual cortex (V1) show increased firing rates and enhanced gain associated with higher arousal (Niell and Stryker, 2010). Interestingly, these state-dependent changes in cor-

\footnotetext{
Received Aug. 24, 2016; revised Oct. 13, 2016; accepted Oct. 19, 2016 The author declares no competing financial interests.

Correspondence should be addressed to Dr. Antoine Barbot, University of Rochester Medical Center and Center for Visual Science, University of Rochester, 601 Elmwood Avenue, Box 314, Rochester, NY 14642. E-mail: antoine_barbot@urmc.rochester.edu.

DOI:10.1523/JNEUROSCI.2691-16.2016

Copyright $\odot 2016$ the authors $\quad 0270-6474 / 16 / 3612080-03 \$ 15.00 / 0$
}

tical activity are reminiscent of the effects of attention in primates (Carrasco, 2011; Harris and Thiele, 2011), suggesting that locomotion and increased alertness in mice may represent a simple model to study changes in cortical activity and sensory representations with attention (Harris and Thiele, 2011).

A recent study by Mineault et al. (2016) furthered this line of research by investigating whether and how locomotion in mice affects spatial resolution, the ability to discriminate fine patterns. Enhanced resolution during locomotion in mice could represent a counterpart to the attentional enhancement of spatial resolution observed in primates (Anton-Erxleben and Carrasco, 2013; Carrasco and Barbot, 2014). Support for enhanced resolution with attention comes from human studies using various psychophysical tasks, such as visual search, acuity, crowding, and texture segmentation (Carrasco and Barbot, 2014). Based on numerous behavioral findings, Carrasco and colleagues (Yeshurun and Carrasco, 1998, 2000; Carrasco et al., 2006; Carrasco and Barbot, 2014; Barbot and Carrasco, 2016) have proposed that attention enhances resolution by preferentially increasing the sensitivity of neurons tuned to high spatial frequencies.

To test this hypothesis, Mineault et al. (2016) measured state-dependent changes in neural sensitivity to, and selectivity for, different spatial frequencies in mouse V1 using two-photon microscopy. Head-fixed mice were allowed to run on a freely rotating platform, spontaneously transitioning between states of low arousal (rest, constricted pupil) and high arousal (locomotion, dilated pupil). Importantly, mice saw a continuous 20-min-long sequence of high-contrast-oriented gratings with random spatial frequencies varying at $4 \mathrm{~Hz}$. The authors tested stimuli over the mouse's spatial-frequency range $(0-0.15$ cycle/deg) (Niell, 2015) to assess neural spatialfrequency tuning and characterize statedependent changes in spatial-frequency representations.

Consistent with previous findings (Niell and Stryker, 2010; McGinley et al., 2015), all V1 neurons showed increased firing rates and enhanced gain during locomotion, and all stimuli were decoded more accurately from population activity. In other words, when the mouse was moving and alert, visual stimuli were better represented. Critically, this gain enhancement was not uniform across spatial-frequency-sensitive neurons. Indeed, the relative gain change of a neuron, defined as the ratio between the gain during locomotion and rest, was tightly related to the neuron's preferred spatial frequency. Neurons tuned to high spatial frequency exhibited larger $(\sim 26 \%)$ gain changes than low-spatial-frequency neurons. This preferential gain enhancement substantially improved the ability to decode high-spatial-frequency information, supporting heightened resolution during locomotion. Importantly, this finding may 


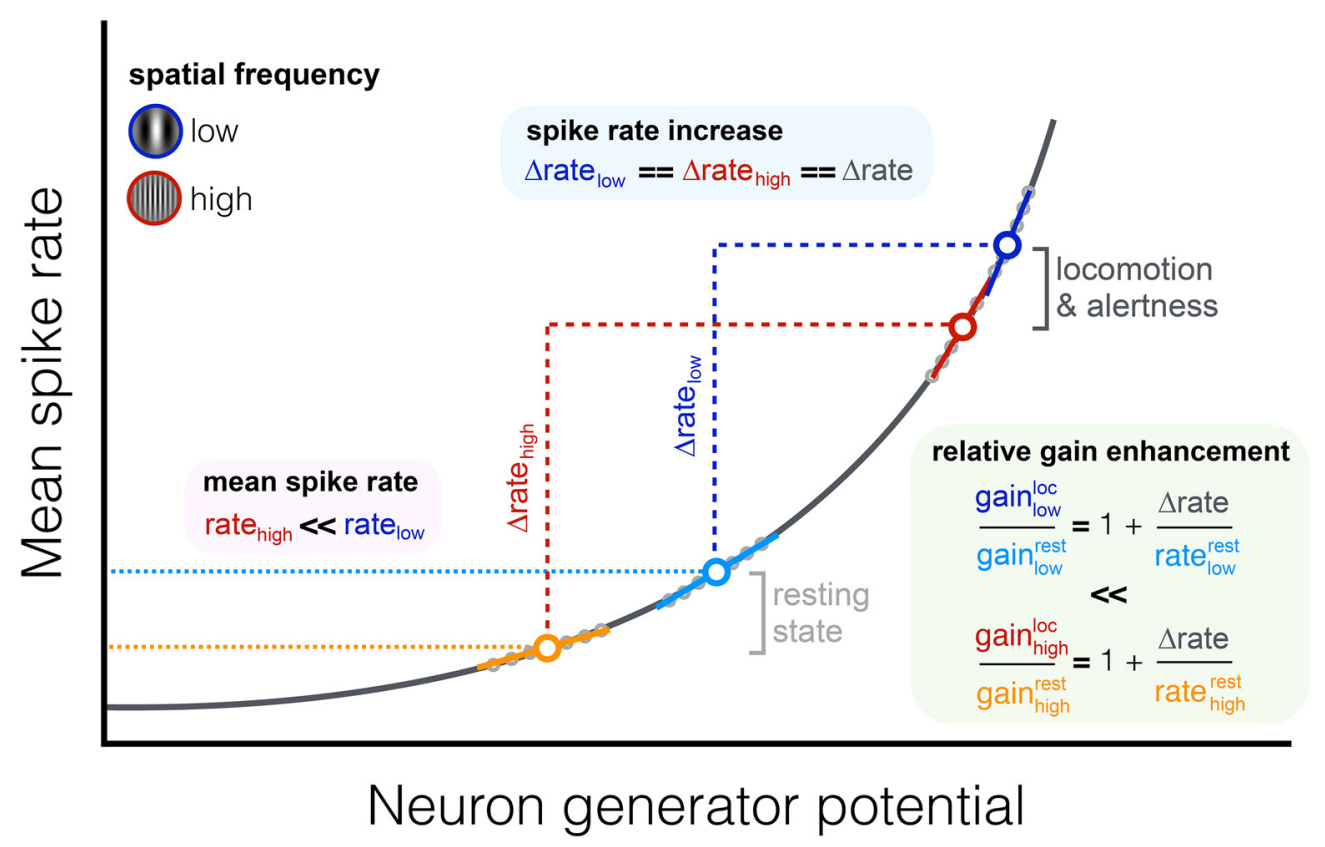

Figure 1. Spike generation is controlled by a generator potential ( $x$-axis) along a spiking nonlinearity, resulting in mean firing rates being proportional to the gain, which is represented by the slope of the linear fits (solid lines). Low- and high-spatial-frequency neurons (dots) exhibited a similar firing rate increase during locomotion ( $\Delta$ rate $_{\text {high }}==\Delta$ rate $_{\text {low }}$ ) but had different operating points (rate high $_{\text {i }} \ll$ rate $_{\text {low }}$ ). Given this difference, a shift in the operating point of $\mathrm{V} 1$ neurons along the spiking nonlinearity between rest and locomotion preferentially enhanced the relative gain of high-spatial-frequency neurons, heightening resolution.

provide neurophysiological evidence that attention enhances resolution by preferentially increasing the gain of high-spatialfrequency neurons, a mechanism first proposed by Carrasco and colleagues (Yeshurun and Carrasco, 1998, 2000; Carrasco et al., 2006; Carrasco and Barbot, 2014; Barbot and Carrasco, 2016).

Another key finding of Mineault et al. (2016) is that this preferential gain change between rest and locomotion originated from shifts in the operating point of neurons (Fig. 1), that is, in the dynamic range of inputs over which neurons respond. The operating point is defined by the neuron's generator potential that controls spike generation along a spiking nonlinearity and represents overall neuronal drive (Ringach and Malone, 2007). The authors found that mean firing rates were proportional to the gain along a spiking nonlinearity. During locomotion, low- and high-spatial-frequency neurons showed similar increases in firing rates. However, high-spatial-frequency neurons had lower rates overall relative to low-spatialfrequency neurons, indicating differences in their operating points. Given this difference, a shift in the operating point of spatialfrequency neurons between rest and locomotion along the spiking nonlinearity could explain the preferential gain enhancement of high-spatial-frequency neurons. This is a novel and interesting idea, but from an evolutionary perspective, it is unclear why hav- ing neural populations with unequal firing rates across preferred features would be beneficial. Further studies are warranted to assess how such differences in operating points arise and whether a similar coupling between the operating point of a neuron and its preferred spatial frequency exists in primates.

The study by Mineault et al. (2016) might play a major role in linking the effects of attention at behavioral and neurophysiological levels, and could help refine current models of attention (Anton-Erxleben and Carrasco, 2013). Neurophysiological studies in primates have found that attention modulates neuronal responses and alters receptive-field properties (Anton-Erxleben and Carrasco, 2013). Specifically, attention enhances neuronal gain and concentrates resources at the attended area by shifting receptive-field centers toward the attentional focus (Connor et al., 1997; Womelsdorf et al., 2006; Anton-Erxleben, Stephan and Treue, 2009). Moreover, attention shrinks receptive-field size (Womelsdorf et al., 2006; Anton-Erxleben et al., 2009), narrowing spatial integration and potentially enhancing resolution. Anton-Erxleben and Carrasco (2013) proposed a linking hypothesis based on the combination of receptivefield shifting and shrinkage in higher-level areas (e.g., V4 and MT) to account for heightened resolution with attention. Future investigations should assess whether enhanced resolution during locomotion in mice is associated with similar receptivefield changes, and relate such changes to Mineault et al. (2016)'s findings in V1.

In interpreting the results of Mineault et al. (2016), however, one should keep in mind some important limitations of the mouse model. First, because of the absence of fovea, mice have uniformly poor acuity vision resembling peripheral vision in primates (Huberman and Niell, 2011; Niell, 2015). Conversely, in primates, resolution is maximal at the fovea and declines with eccentricity, with the neurons' preferred spatial frequency shifting from high to low with eccentricity (Anton-Erxleben and Carrasco, 2013). Given that the effects of attention do not decline with eccentricity (Carrasco and Barbot, 2014), the coupling between a neuron's preferred spatial frequency and its operating point might depend on eccentricity in primates. One may assume that the operating point of a neuron depends not only on its preferred spatial frequency, but also on the preferred spatial-frequency range of the population at a given eccentricity. Based on Mineault et al. (2016), subsets of neurons tuned to higher spatial frequencies within the population should be preferentially enhanced relative to lowerspatial-frequency neurons. Therefore, a larger gain enhancement of neurons tuned to the highest spatial frequency at a given eccentricity would result in similar resolution enhancement across eccentric- 
ities, despite differences in spatial-frequency preferences with eccentricity.

Second, mice offer no counterpart of the spatial allocation of attention in primates. Spatial attention alters processing at both attended and unattended areas, enhancing contrast sensitivity and spatial resolution at attended areas at the expense of decreased sensitivity and poorer resolution at unattended areas (Carrasco, 2011). The findings of Mineault et al. (2016) can explain the effects of attention at attended areas. By analogy, a preferential gain reduction of high-spatial-frequency neurons at unattended areas may account for impaired sensitivity and poorer resolution.

Furthermore, increasing resolution can sometimes be detrimental (e.g., when low spatial frequencies are most informative or when a global visual assessment is required). Human studies have shown that exogenous (involuntary, stimulus-driven) attention automatically enhances resolution even when detrimental for the task at hand, and does so by enhancing high spatial frequencies (Yeshurun and Carrasco, 1998, 2000; Carrasco et al., 2006). Conversely, endogenous (voluntary, goal-driven) attention can flexibly adjust its operation on resolution and optimize performance according to task demands (Yeshurun et al., 2008; Barbot and Carrasco, 2016). Unfortunately, the mouse model turns out to be inadequate with respect to these findings.

Finally, although locomotion-induced effects in mice share similarities with attentional effects in primates, locomotion cannot simply be associated with increased arousal. For instance, Vinck et al. (2015) dissociated locomotion-specific increases in firing rates from enhanced neural encoding with higher arousal during locomotion. Mineault et al. (2016) speculated that enhanced high-spatial-frequency representations during locomotion might be related to motor signals and serve to compensate for motion blur. In humans, saccade preparation results in a preferential enhancement of high-spatial-frequency representations ( $\mathrm{Li}$ et al., 2016), which is reminiscent of atten- tional effects in primates (Carrasco and Barbot, 2014) and locomotion in mice (Mineault et al., 2016). Given the intermingling of perception and action, future studies will benefit from recent success training mice to report their percept in wellcharacterized psychophysical tasks, analogous to those used in primates (Huberman and Niell, 2011).

Although Mineault et al. (2016) did not directly manipulate attention, their findings provide neurophysiological evidence for the hypothesis that attention enhances spatial resolution by preferentially enhancing the sensitivity of high-spatial-frequency neurons (Carrasco and Barbot, 2014). Mice have emerged as a powerful model system for studying state-dependent changes in cortical activity (Harris and Thiele, 2011), helping bridge the gap between molecular and cellular changes in cortical circuits and perceptual representations. Together, these studies portray the brain as a dynamic system in which state-dependent variations fine-tune neuronal gain and shape our perceptual experience on a moment-tomoment basis.

\section{References}

Anton-Erxleben K, Carrasco M (2013) Attentional enhancement of spatial resolution: linking behavioural and neurophysiological evidence. Nat Rev Neurosci 14:188-200. CrossRef Medline

Anton-Erxleben K, Stephan VM, Treue S (2009) Attention reshapes center-surround receptive field structure in macaque cortical area MT. Cereb Cortex 19:2466-2478. CrossRef Medline

Barbot A, Carrasco M (2016) Attention modifies spatial resolution according to task demands. Psychol Sci, in press.

Carrasco M (2011) Visual attention: the past 25 years. Vision Res 51:1484-1525. CrossRef Medline

Carrasco M, Barbot A (2014) How attention affects spatial resolution. Cold Spring Harb Symp Quant Biol 79:149-160. CrossRef Medline

Carrasco M, Loula F, Ho YX (2006) How attention enhances spatial resolution: evidence from selective adaptation to spatial frequency. Percept Psychophys 68:1004-1012. CrossRef Medline
Connor CE, Preddie DC, Gallant JL, Van Essen DC (1997) Spatial attention effects in macaque area V4. J Neurosci 17:3201-3214. Medline

Harris KD, Thiele A (2011) Cortical state and attention. Nat Rev Neurosci 12:509-523. CrossRef Medline

Huberman AD, Niell CM (2011) What can mice tell us about how vision works? Trends Neurosci 34:464-473. CrossRef Medline

Li HH, Barbot A, Carrasco M (2016) Saccade preparation reshapes sensory tuning. Curr Biol 26:1564-1570. CrossRef Medline

McGinley MJ, Vinck M, Reimer J, Batista-Brito R, Zagha E, Cadwell CR, Tolias AS, Cardin JA, McCormick DA (2015) Waking state: rapid variations modulate neural and behavioral responses. Neuron 87:1143-1161. CrossRef Medline

Mineault PJ, Tring E, Trachtenberg JT, Ringach DL (2016) Enhanced spatial resolution during locomotion and heightened attention in mouse primary visual cortex. J Neurosci 36 : 6382-6392. CrossRef Medline

Niell CM (2015) Cell types, circuits, and receptive fields in the mouse visual cortex. Annu Rev Neurosci 38:413-431. CrossRef Medline

Niell CM, Stryker MP (2010) Modulation of visual responses by behavioral state in mouse visual cortex. Neuron 65:472-479. CrossRef Medline

Ringach DL, Malone BJ (2007) The operating point of the cortex: neurons as large deviation detectors. J Neurosci 27:7673-7683. CrossRef Medline

Vinck M, Batista-Brito R, Knoblich U, Cardin JA (2015) Arousal and locomotion make distinct contributions to cortical activity patterns and visual encoding. Neuron 86:740-754. CrossRef Medline

Womelsdorf T, Anton-Erxleben K, Pieper F, Treue S (2006) Dynamic shifts of visual receptive fields in cortical area MT by spatial attention. Nat Neurosci 9:1156-1160. CrossRef Medline

Yeshurun Y, Carrasco M (1998) Attention improves or impairs visual performance by enhancing spatial resolution. Nature 396:72-75. CrossRef Medline

Yeshurun Y, Carrasco M (2000) The locus of attentional effects in texture segmentation. Nat Neurosci 3:622-627. CrossRef Medline

Yeshurun Y, Montagna B, Carrasco M (2008) On the flexibility of sustained attention and its effects on a texture segmentation task. Vision Res 48:80-95. CrossRef Medline 